\title{
SCHURITY AND SEPARABILITY OF QUASIREGULAR COHERENT CONFIGURATIONS
}

\author{
MITSUGU HIRASAKA, KIJUNG KIM, AND ILIA PONOMARENKO
}

\begin{abstract}
A permutation group is said to be quasiregular if every its transitive constituent is regular, and a quasiregular coherent configuration can be thought as a combinatorial analog of such a group: the transitive constituents are replaced by the homogeneous components. In this paper, we are interested in the question when the configuration is schurian, i.e., formed by the orbitals of a permutation group, or/and separable, i.e., uniquely determined by the intersection numbers. In these terms, an old result of Hanna Neumann is, in a sense, dual to the statement that the quasiregular coherent configurations with cyclic homogeneous components are schurian. In the present paper, we (a) establish the duality in a precise form and (b) generalize the latter result by proving that a quasiregular coherent configuration is schurian and separable if the groups associated with homogeneous components have distributive lattices of normal subgroups.
\end{abstract}

\section{INTRODUCTION}

A permutation group is said to be quasiregular if every its transitive constituent is regular [1, p. 53]. This concept has an obvious direct analog in the theory of "groups without groups": a coherent configuration is said to be quasiregular if each its homogeneous component is regular (for exact definitions concerning coherent configurations, see Section 22. In fact, there is a one-to-one correspondence between the regular groups (which are exactly transitive quasiregular groups) and regular coherent configurations 11. However, no such a correspondence exists for intransitive quasiregular groups: the reason is that not every quasiregular coherent configuration is schurian, i.e., formed by the orbitals of a permutation group. The first example of a non-schurian quasiregular coherent configuration was constructed by Sergei Evdokimov in the end of 1990s (but was never published). One of motivations for the present paper is to find conditions for a quasiregular coherent configuration to be schurian.

Another motivation comes from an old paper of Hanna Neumann [10] 1 where she studied the amalgams of finite cyclic groups. Roughly speaking, the question

Date: December 31, 2017.

This research was supported by Basic Science Research Program through the National Research Foundation of Korea(NRF) funded by the Ministry of Science, ICT \& Future Planning(NRF2016R1A2B4013474).

The second author was supported by Basic Science Research Program through the National Research Foundation of Korea funded by the Ministry of Education (NRF-2017R1D1A3B03031349).

The work of the third author was supported by the RAS Program of Fundamental Research "Modern Problems of Theoretical Mathematics".

${ }^{1}$ The third author is expressed gratitude to Mikhail Muzychuk, who drew his attention to paper [10]. 
is whether a family $\mathfrak{G}$ of groups with prescribed pairwise intersections admits an amalgam, i.e., can be isomorphically embedded to a certain group. The main result of [10] states that if $\mathfrak{G}$ consists of finite cyclic groups, then this is true if some natural necessary conditions concerning the pairwise intersections are satisfied. These conditions have sense not only for cyclic groups and for any family $\mathfrak{G}$ satisfying these conditions, we use term amalgam configuration based on $\mathfrak{G}$ (the exact definition is given in Section 3). Not every amalgam configuration based on a family $\mathfrak{G}$ of abelian groups admits an amalgam. A reason for this is revealed in the theorem below establishing a close relationship between the amalgam configurations based on $\mathfrak{G}$ and quasiregular coherent configuration of type $\mathfrak{G}$, i.e., those the homogeneous components of which are exactly the groups from $\mathfrak{G}$.

Theorem 1.1. Let $\mathfrak{G}$ be a family of finite abelian groups. Then the quasiregular coherent configurations of type $\mathfrak{G}$ are in one-to-one correspondence with the amalgam configurations based on $\mathfrak{G}$. Moreover, a coherent configuration of type $\mathfrak{G}$ is schurian if and only if the corresponding amalgam configuration admits an amalgam.

The proof of Theorem 1.1 is presented in Section 5 and uses a quite general concept of a system of linked sections based on a family $\mathfrak{G}$ of groups (see Section 3). The special cases of this concept are the amalgam configurations and quasiregular coherent configurations; the fact that the latter are in one-to-one correspondence with the systems of linked quotients is proved in Section 4 . The main idea of the whole proof is to show that if the family $\mathfrak{G}$ consists of abelian groups, then these two special cases are dual each to other in the sense of the duality theory of abelian groups.

From Theorem 1.1] it immediately follows that the above mentioned result of Hanna Neumann is equivalent to the fact that any quasiregular coherent configuration such that all its homogeneous components are cyclic, is schurian. Since the subgroup lattice of a cyclic group is distributive, Theorem 1.2 below generalizes the latter statement to a broader class of groups containing all cyclic groups. Before we state this result in a precise form, we discuss one more concept making this theorem even more stronger.

Let $K \leq \operatorname{Sym}(\Omega)$, and let $\mathcal{X}$ be the coherent configuration formed by the orbitals of $K$. A natural invariant of $\mathcal{X}$ and hence of $K$ is given by the tensor of intersection numbers: they can be thought as the structure constants of the the centralizer algebra of $K$ with respect to the linear basis formed by the adjacency matrices of the orbitals of $K$. The question going back to D. Higman [7] is when this tensor determines the group $K$ up to permutation isomorphism. In the language of the coherent configurations, the positive answer exactly means that $\mathcal{X}$ is separable. Now, we are ready to state the second main result of the present paper.

Theorem 1.2. Let $\mathfrak{G}$ be a family of groups with distributive lattices of normal subgroups. Then any quasiregular coherent configuration of type $\mathfrak{G}$ is schurian and separable.

We deduce this result from Theorem 6.2 proved in Section 6 for even more broader class of coherent configurations. The idea of the proof is close to that used in P. H. Zieschang's paper [12, where the schurity part of Theorem 1.2 was proved for a class of homogeneous coherent configurations. Note that these configurations 
are nothing else than homogeneous algebraic fusions of quasiregular coherent configuration of type $\mathfrak{G}$ satisfying the condition of Theorem 1.2 and such that all the groups in $\mathfrak{G}$ are isomorphic.

In Section 7, we study the schurity and separability of all quasiregular coherent configurations with small number of homogeneous components. The main result here is presented by the following theorem.

Theorem 1.3. A quasiregular coherent configuration with at most three homogeneous components is schurian and separable.

In the case of commutative homogeneous components, the schurity part of Theorem 1.3 was proved in 6 . In Section 7 we also construct an infinite family of non-schurian quasiregular coherent configurations with four commutative homogeneous components. The question of separability of these coherent configurations remains open.

The class of meta-thin association schemes was introduced and studied by the first author in 8. From [4, Theorem 2.1], it follows that any such scheme is an algebraic fusion of a quasiregular coherent configuration with isomorphic homogeneous components. The number of them equals the index of the thin residue of the original association scheme. Thus, as a corollary of Theorem 1.3 we obtain the following statement also proved in Section 7 .

Corollary 1.4. Any meta-thin scheme with thin residue of index at most three is schurian and separable.

Notation.

Throughout the paper, $\Omega$ denotes a finite set.

The diagonal of the Cartesian product $\Omega \times \Omega$ is denoted by $1_{\Omega}$.

For a relation $r \subseteq \Omega \times \Omega$, we set $r^{*}=\{(\beta, \alpha):(\alpha, \beta) \in r\}$ and $\alpha r=\{\beta \in \Omega$ : $(\alpha, \beta) \in r\}$ for all $\alpha \in \Omega$.

For a relation $r \subseteq \Omega \times \Omega$ and sets $\Delta, \Gamma \subseteq \Omega$, we set $s_{\Delta, \Gamma}=s \cap(\Delta \times \Gamma)$. If $S$ is a set of relations, we put $S_{\Delta, \Gamma}=\left\{s_{\Delta, \Gamma}: s \in S\right\}$ and denote $S_{\Delta, \Delta}$ by $S_{\Delta}$.

For relations $r, s \subseteq \Omega \times \Omega$, we set $r \cdot s=\{(\alpha, \beta):(\alpha, \gamma) \in r,(\gamma, \beta) \in s$ for some $\gamma \in \Omega\}$. If $S$ and $T$ are sets of relations, we set $S \cdot T=\{s \cdot t: s \in S, t \in T\}$.

For a set $S$ of relations on $\Omega$, we denote by $S^{\cup}$ the set of all unions of the elements of $S$, and put $S^{*}=\left\{s^{*}: s \in S\right\}$ and $\alpha S=\cup_{s \in S} \alpha s$, where $\alpha \in \Omega$.

\section{Coherent configurations}

In our presentation of coherent configurations, we follow papers 9,3 and monograph [11. All the facts we use, can be found in these sources and references therein.

2.1. Definitions. A pair $\mathcal{X}=(\Omega, S)$, where $\Omega$ is a finite set and $S$ is a partition of $\Omega \times \Omega$, is called a coherent configuration on $\Omega$ if $1_{\Omega} \in S^{\cup}, S^{*}=S$, and if given $r, s, t \in S$, the number

$$
c_{r s}^{t}=\left|\alpha r \cap \beta s^{*}\right|
$$

\footnotetext{
${ }^{2}$ Theorem 5.6 in [6] states that any quasiregular coherent configuration with commutative homogeneous components is separable. However, this is not true: an infinite family of counterexamples was constructed in [2] Section 5].
} 
does not depend on a choice of $(\alpha, \beta) \in t$. The elements of $\Omega, S, S^{\cup}$, and the numbers $c_{r s}^{t}$ are called the points, basis relations, relations and intersection numbers of $\mathcal{X}$, respectively. The numbers $|\Omega|$ and $|S|$ are called the degree and the rank of $\mathcal{X}$. A unique basic relation containing a pair $(\alpha, \beta) \in \Omega \times \Omega$ is denoted by $r(\alpha, \beta)$. Since the mapping $r: \Omega \times \Omega \rightarrow S$ depends only on $\mathcal{X}$, it should be denoted by $r_{\mathcal{X}}$, but we usually omit the subindex if this does not lead to confusion.

The set $S^{\cup}$ contains the relation $r \cdot s$ for all $r, s \in S^{\cup}$. It follows that this relation is the union (possibly empty) of basis relations of $\mathcal{X}$; the set of these relations is called the complex product of $r$ and $s$ and denoted by $r s$. Thus

$$
r s \subseteq S, \quad r, s \in S .
$$

In what follows, for any $X, Y \subseteq S$, we denote by $X Y$ the union of all sets $r s$ with $r \in X$ and $s \in Y$. Obviously, $(X Y) Z=X(Y Z)$ for all $X, Y, Z \subseteq S$. A nonempty subset $X$ of $S$ is said to be closed if $X X^{*} \subseteq X$.

2.2. Fibers and homogeneity. A set $\Delta \subseteq \Omega$ is called a fiber of the coherent configuration $\mathcal{X}$ if $1_{\Delta} \in S$; the set of all fibers is denoted by $F=F(\mathcal{X})$. The point set $\Omega$ is a disjoint union of fibers. If $\Delta$ is a union of fibers, then the pair

$$
\mathcal{X}_{\Delta}=\left(\Delta, S_{\Delta}\right)
$$

is a coherent configuration, called the restriction of $\mathcal{X}$ to $\Delta$; it is called a homogeneous component of $\mathcal{X}$ if $\Delta \in F$. The coherent configuration is said to be homogeneous or association scheme or scheme if it has exactly one homogeneous component, or equivalently if $1_{\Omega} \in S$.

For any basic relation $s \in S$, there exist uniquely determined fibers $\Delta, \Gamma$ such that $s \subseteq \Delta \times \Gamma$; in particular, the union

$$
S=\bigcup_{\Delta, \Gamma \in F} S_{\Delta, \Gamma}
$$

is disjoint. The number $|\delta s|$ with $\delta \in \Delta$, equals the intersection number $c_{s s^{*}}^{1 \Delta}$, and hence does not depend on the choice of the point $\delta$. It is called the valency of $s$ and denoted by $n_{s}$. For each fiber $\Delta$ and $s \in S_{\Delta}$ we have $n_{s}=n_{s^{*}}$.

2.3. Isomorphisms and schurity. Two coherent configurations are called isomorphic if there exists a bijection between their point sets that induces the bijection between their sets of basis relations. Each such bijection is called an isomorphism between these two configurations. The group of all isomorphisms of a coherent configuration $\mathcal{X}=(\Omega, S)$ to itself contains a normal subgroup

$$
\operatorname{Aut}(\mathcal{X})=\left\{f \in \operatorname{Sym}(\Omega): s^{f}=s, s \in S\right\}
$$

called the automorphism group of $\mathcal{X}$, where $s^{f}=\left\{\left(\alpha^{f}, \beta^{f}\right):(\alpha, \beta) \in s\right\}$.

Conversely, let $K \leq \operatorname{Sym}(\Omega)$ be a permutation group, and let $S=\operatorname{Orb}\left(K, \Omega^{2}\right)$. Then, $\mathcal{X}=(\Omega, S)$ is a coherent configuration; we say that $\mathcal{X}$ is associated with $K$. A coherent configuration on $\Omega$ is said to be schurian if it is associated with some permutation group on $\Omega$. A coherent configuration $\mathcal{X}$ is schurian if and only if it is associated with the group $\operatorname{Aut}(\mathcal{X})$. 
2.4. Algebraic isomorphisms and separability. Let $\mathcal{X}=(\Omega, S)$ and $\mathcal{X}^{\prime}=$ $\left(\Omega^{\prime}, S^{\prime}\right)$ be coherent configurations. A bijection $\varphi: S \rightarrow S^{\prime}, r \mapsto r^{\prime}$ is called an algebraic isomorphism from $\mathcal{X}$ onto $\mathcal{X}^{\prime}$ if

$$
c_{r s}^{t}=c_{r^{\prime} s^{\prime}}^{t^{\prime}}, \quad r, s, t \in S .
$$

In this case, $\mathcal{X}$ and $\mathcal{X}^{\prime}$ are said to be algebraically isomorphic. Each isomorphism $f$ from $\mathcal{X}$ onto $\mathcal{X}^{\prime}$ induces an algebraic isomorphism $\varphi_{f}: r \mapsto r^{f}$ between these configurations. The set of all isomorphisms inducing the algebraic isomorphism $\varphi$ is denoted by $\operatorname{Iso}\left(\mathcal{X}, \mathcal{X}^{\prime}, \varphi\right)$. In particular,

$$
\operatorname{Iso}\left(\mathcal{X}, \mathcal{X}, \operatorname{id}_{S}\right)=\operatorname{Aut}(\mathcal{X})
$$

where $\operatorname{id}_{S}$ is the identity mapping on $S$. A coherent configuration $\mathcal{X}$ is said to be separable if for any algebraic isomorphism $\varphi: S \rightarrow S^{\prime}$, the $\operatorname{set} \operatorname{Iso}\left(\mathcal{X}, \mathcal{X}^{\prime}, \varphi\right)$ is not empty.

The algebraic isomorphism $\varphi$ induces a bijection from $S^{\cup}$ onto $\left(S^{\prime}\right)^{\cup}$ : the union $r \cup s \cup \cdots$ of basis relations of $\mathcal{X}$ is taken to $r^{\prime} \cup s^{\prime} \cup \cdots$. This bijection is also denoted by $\varphi$. One can see that $\varphi$ preserves the reflexive basis relations on all fibers. This extends $\varphi$ to a bijection $F(\mathcal{X}) \rightarrow F\left(\mathcal{X}^{\prime}\right)$ so that $\left(1_{\Delta}\right)^{\prime}=1_{\Delta^{\prime}}$.

2.5. Faithful maps. Let $\mathcal{X}=(\Omega, S)$ and $\mathcal{X}^{\prime}=\left(\Omega^{\prime}, S^{\prime}\right)$ be coherent configurations, and let $\varphi: S \rightarrow S^{\prime}$ be an algebraic isomorphism. A bijection $f$ from a subset of $\Omega$ to a subset of $\Omega^{\prime}$ is said to be $\varphi$-faithful if

$$
r(\alpha, \beta)^{\varphi}=r^{\prime}\left(\alpha^{f}, \beta^{f}\right) \text { for all } \alpha, \beta \in \operatorname{Dom}(f),
$$

where $\operatorname{Dom}(f)$ is the domain of $f$. Note that if this domain is a singleton $\{\alpha\}$ and $\Delta$ is the fiber of $\mathcal{X}$ containing $\alpha$, then the fiber of $\mathcal{X}^{\prime}$ containing $\alpha^{f}$ is equal to $\Delta^{\varphi}$. Clearly, if $f$ is a $\varphi$-faithful map, then the restriction of $f$ to any subset of $\operatorname{Dom}(f)$ is also $\varphi$-faithful.

A $\varphi$-faithful map $f$ is said to be $\varphi$-extendable to a point $\gamma \in \Omega$ if there exists a $\varphi$-faithful map with domain $\operatorname{Dom}(f) \cup\{\gamma\}$, or, equivalently, if

$$
\bigcap_{\alpha \in \operatorname{Dom}(f)} \alpha^{f} r(\alpha, \gamma)^{\varphi} \neq \varnothing \text {. }
$$

A $\varphi$-faithful map which is $\varphi$-extendable to every point of $\Omega$, is said to be $\varphi$ extendable. From the definitions of coherent configurations and algebraic isomorphisms, it follows that every $\varphi$-faithful map $f$ with $|\operatorname{Dom}(f)| \leq 2$ is $\varphi$-extendable. In these terms, one can give a sufficient condition for schurity and separability of a coherent configuration.

Lemma 2.1. Let $\mathcal{X}=(\Omega, S)$ be a coherent configuration. Then

(1) $\mathcal{X}$ is schurian if every $\varphi$-faithful map with $\varphi=\operatorname{id}_{S}$ is $\varphi$-extendable,

(2) $\mathcal{X}$ is separable if for every algebraic isomorphism $\varphi$ from $\mathcal{X}$ onto another coherent configuration, each $\varphi$-faithful map is $\varphi$-extendable.

Proof. Let $\varphi: \mathcal{X} \rightarrow \mathcal{X}^{\prime}$ be an algebraic isomorphism. Assume that each $\varphi$-faithful map is $\varphi$-extendable. Then for any pairs $(\alpha, \beta) \in \Omega \times \Omega$ and $\left(\alpha^{\prime}, \beta^{\prime}\right) \in \Omega^{\prime} \times \Omega^{\prime}$ such that

$$
r(\alpha, \beta)^{\varphi}=r^{\prime}\left(\alpha^{\prime}, \beta^{\prime}\right),
$$

there exists an isomorphism $f \in \operatorname{Iso}\left(\mathcal{X}, \mathcal{X}^{\prime}, \varphi\right)$ taking $\alpha$ to $\alpha^{\prime}$, and $\beta$ to $\beta^{\prime}$. Now if $\mathcal{X}=\mathcal{X}^{\prime}$ and $\varphi$ is the identity algebraic isomorphism, then $f \in \operatorname{Aut}(\mathcal{X})$ and 
the coherent configuration $\mathcal{X}$ is schurian. This proves statement (1). Also, if $\varphi$ runs over all algebraic isomorphisms from $\mathcal{X}$, then $\mathcal{X}$ is separable, which proves statement (2).

Corollary 2.2. Let $\mathcal{X}$ be a coherent configuration. Suppose that for every algebraic isomorphism $\varphi$ onto another coherent configuration, each $\varphi$-faithful map is $\varphi$-extendable. Then $\mathcal{X}$ is schurian and separable.

2.6. Quasiregular coherent configurations. A relation $s \subseteq \Omega \times \Omega$ is said to be thin if $|\alpha s| \leq 1$ and $\left|\beta s^{*}\right| \leq 1$ for all $\alpha, \beta \in \Omega$, or equivalently, $s$ is a bijection from $\operatorname{Dom}(s)$ onto $\operatorname{Dom}\left(s^{*}\right)$. In the latter sense, $s^{*}$ is the bijection inverse to $s$. One can easily see that if $\mathcal{X}=(\Omega, S)$ is a coherent configuration and $s \in S$, then $n_{s}=n_{s^{*}}=1$ and $s \cdot r, r \cdot s \in S$ for all $r \in S$ unless $s \cdot r$ or $s \cdot r$ are empty.

Lemma 2.3. Let $\mathcal{X}=(\Omega, S)$ be a coherent configuration and $s \in S$. Assume that the set $s s^{*}$ consists of thin relations. Then $s s^{*} s=\{s\}$.

Proof. Let $t \in s s^{*}$. Then $t$ is a thin relation by the assumption and hence $t s$ is a singleton. Since also $s \in t s$, it follows that $t s=\{s\}$. This is true for all $t \in s s^{*}$ and hence $s s^{*} s \subseteq\{s\}$. However, $1_{\Delta} \in s s^{*}$, where $\Delta$ is a unique fiber of $\mathcal{X}$ such that $s \subseteq \Delta \times \Omega$. Thus, $s=1_{\Delta} \cdot s \in s s^{*} s$ and so $s s^{*} s=\{s\}$.

A coherent configuration $\mathcal{X}$ is said to be semiregular if every its basis relation is thin. If, in addition, it is homogeneous, then we say that $\mathcal{X}$ is a regular scheme (or thin scheme in the terminology of [12]). Finally, $\mathcal{X}$ is said to be quasiregular if each homogeneous component of $\mathcal{X}$ is regular. Note that if $K$ is a permutation group and $\mathcal{X}$ is the coherent configuration associated with $K$, then $\mathcal{X}$ is semiregular (respectively, regular, quasiregular) if and only if the group $K$ is semiregular (respectively, regular, quasiregular). It should also be noted that the basis relations of a regular scheme form a group with respect to the product · of relations.

\section{Systems OF LINKED SECTIONS}

In this section, we introduce an auxiliary structure, which enables us to deal with quasiregular coherent configurations and generalized free products in a uniform way. To do this, we define the intersection of two sections $S=U / L$ and $S^{\prime}=U^{\prime} / L^{\prime}$ of a group $G$ by the formula

$$
S \cap S^{\prime}= \begin{cases}\left(U \cap U^{\prime}\right) / L L^{\prime} & \text { if } L L^{\prime} \leq U \cap U^{\prime} \\ \varnothing & \text { otherwise. }\end{cases}
$$

Note that the intersection is nonempty if $U=U^{\prime}=G$ or $L=L^{\prime}=1$. We say that $S$ is a subsection of $S^{\prime}$ if $L^{\prime} \leq L$ and $U \leq U^{\prime}$; in this case, we use notation $S \prec S^{\prime}$. Clearly, the nonempty intersection $S \cap S^{\prime}$ is a subsection of both $S$ and $S^{\prime}$.

Let $I$ be a finite set, and let

(F1) $\mathfrak{G}$ be a family of finite groups $G_{i}$ with identity $e_{i}$,

(F2) $\mathfrak{S}$ be a family of sections $S_{i j}$ of $G_{i}$, where $S_{i i}=G_{i} / e_{i}$,

(F3) $\mathfrak{F}$ be a family of isomorphisms $f_{i j} \in \operatorname{Iso}\left(S_{i j}, S_{j i}\right)$, where $f_{i i}=f_{i j} f_{j i}=\mathrm{id}$, where the indices $i$ and $j$ run over $I$. We assume that

$$
S_{i j} \cap S_{i k} \neq \varnothing, \quad i, j, k \in I .
$$

This enables us to define the section $S_{i j k}=S_{i j} \cap S_{i k}$ of the group $G_{i}$; it is easily seen that $S_{i j k}=S_{i k j}$ for all $i, j, k$, and $S_{i j k}=S_{i j}$ whenever $k=i$ or $k=j$. The 
isomorphism $f_{i k}$ takes the subsections of $S_{i k}$ to those of $S_{k i}$, and also preserves the partial order $\prec$. Therefore, $f_{i k}\left(S_{i j k}\right) \prec f_{i k}\left(S_{i k}\right)=S_{k i}$. We assume that

$$
f_{i k}\left(S_{i j k}\right)=S_{k i j}, \quad i, j, k \in I .
$$

Thus, the isomorphism $f_{i k}$ induces an isomorphism from $S_{i j k}$ onto $S_{k i j}$; we denote it by $f_{i j k}$.

Definition 3.1. The triple $(\mathfrak{G}, \mathfrak{S}, \mathfrak{F})$ defined by $(\mathrm{F} 1),(\mathrm{F} 2)$, and $(\mathrm{F} 3)$ is called a system of linked sections (based on $\mathfrak{G}$ ) if, in addition to conditions (4) and (5),

$$
f_{j k i} f_{k i j} f_{i j k}=\mathrm{id}, \quad i, j, k \in I .
$$

The two special cases we are interested in are the systems of linked quotients and linked subgroups. In the former case, $S_{i j}=G_{i} / L_{i j}$ for all $i, j$; in particular, $L_{i j}$ is a normal subgroup of $G_{i}$. In the latter case $S_{i j}=U_{i j} / e_{i}$ for all $i, j$; in this case, $S_{i j}$ is identified with a subgroup of $G_{i j}$. As we will see in Section 4 a systems of linked quotients is nothing else than a quasiregular coherent configuration.

Let $(\mathfrak{G}, \mathfrak{S}, \mathfrak{F})$ be a system of linked subgroups. In this case, $S_{i j}$ is identified with a subgroup $U_{i j}$ of the group $G_{i}$, and also $U_{i}:=U_{i i}$ is equal to $G_{i}, i, j \in I$. Assume that all the groups $U_{i}$ are abelian. Then the direct product

$$
U=\prod_{i \in I} U_{i}
$$

is also abelian, and coincides with the free product of the groups $U_{i}$. It contains a subgroup

$$
U_{0}=\left\langle x^{-1} f_{i j}(x): x \in U_{i j}, i, j \in I\right\rangle,
$$

where $f_{i j}: U_{i j} \rightarrow U_{j i}$ is the isomorphism in (F3). Let

$$
\pi: U \rightarrow U / U_{0}
$$

be the canonical epimorphism. In this notation, the group $\pi(U)$ is called the generalized free product of the groups $U_{i}$ with amalgamated subgroups $U_{i j}$ if

$$
\pi\left(U_{i}\right) \cong U_{i} \quad \text { and } \quad \pi\left(U_{i}\right) \cap \pi\left(U_{j}\right)=\pi\left(U_{i j}\right), \quad i, j \in I,
$$

see paper [10, p. 672]. It was remarked there that formulas (6) form a necessary condition for existence of the generalized free product of the groups $A_{i}$ with amalgamated subgroups $A_{i j}$. In this sense, the system $(\mathfrak{G}, \mathfrak{S}, \mathfrak{F})$ of linked subgroups will also be called an amalgam configuration (based on $\mathfrak{G}$ ), and we say that it admits an amalgam if the generalized free product of the groups $U_{i}$ with amalgamated subgroups $U_{i j}$ does exists, i.e., condition (7) is satisfied.

Duality for systems of linked sections. The aim of this part is to define a dual of a system of linked sections based on a family of abelian groups. To this end, we recall some standard facts from the duality theory for abelian groups and fix notations.

For an abelian group $G$, we denote by $\widehat{G}$ its dual group, i.e. the group of complexvalued characters of $G$ with respect to componentwise multiplication. There is a canonical mapping from the subgroup lattice of $G$ to that of $\widehat{G}$, taking a group $H \leq G$ to the group

$$
H^{\perp}=\{\chi \in \widehat{G}: H \subseteq \operatorname{ker}(\chi)\}
$$


which is isomorphic to the dual group of $G / H$. Identifying the group $G$ with the dual group of $\widehat{G}$, we have $\left(H^{\perp}\right)^{\perp}=H$. The mapping $\perp$ is an anti-isomorphism of the subgroup lattices. Namely, for all subgroups $L$ and $U$ of the group $G$,

$$
L \leq U \quad \Leftrightarrow \quad U^{\perp} \leq L^{\perp}
$$

and

$$
(L U)^{\perp}=L^{\perp} \cap U^{\perp} \quad \text { and } \quad(L \cap U)^{\perp}=L^{\perp} U^{\perp} .
$$

We extend the mapping $\perp$ to the sections of $G$ by setting $(U / L)^{\perp}=L^{\perp} / U^{\perp}$. Clearly, this group is canonically isomorphic to the dual group of $U / L$. It is a routine to check that for any two sections $S$ and $T$ of the group $G$, we have $S \cap T \neq \varnothing$ if and only if $S^{\perp} \cap T^{\perp} \neq \varnothing$, and in this case

$$
(S \cap T)^{\perp}=S^{\perp} \cap T^{\perp} .
$$

Now let $U$ and $L$ be arbitrary subgroups of the group $G$, and let $\pi: U \rightarrow L$ a group homomorphism. Then one can define the mapping $\pi^{\perp}: L^{\perp} \rightarrow U^{\perp}$ that takes the character $\chi \in L^{\perp}$ to the character $\chi^{\pi^{\perp}} \in U^{\perp}$ defined by the formula

$$
\chi^{\pi^{\perp}}(u)=\chi(\pi(u)), \quad u \in U .
$$

One can see that the mapping $\pi \mapsto \pi^{\perp}$ is a group isomorphism from $\operatorname{Hom}(U, L)$ onto $\operatorname{Hom}\left(L^{\perp}, U^{\perp}\right)$ that preserves isomorphisms. It is routine to define the isomorphism $\pi^{\perp}: T^{\perp} \rightarrow S^{\perp}$ for any isomorphism $\pi: S \rightarrow T$, where $S$ and $T$ are the sections of $G$.

Let $(\mathfrak{G}, \mathfrak{S}, \mathfrak{F})$ be a system of linked sections such that the family $\mathfrak{G}$ consists of abelian groups. Denote by $A$ the group dual to the group $G=\prod_{i \in I} G_{i}$. Then using formulas (9), one can see that $A$ is the direct product of the groups

$$
A_{i}=G_{i^{\prime}}^{\perp}, \quad i \in I,
$$

where $G_{i^{\prime}}$ is the direct product of the $G_{j}$ with $j \neq i$. Assume that $S_{i j}=U_{i j} / L_{i j}$, where $U_{i j}$ and $L_{i j}$ are subgroups of $G_{i} \leq G$, and also $L_{i j} \leq U_{i j}$. Then by formulas (91), the group

$$
A_{i j}:=\left(\left(G_{i^{\prime}} U_{i j}\right) /\left(G_{i^{\prime}} L_{i j}\right)\right)^{\perp}=\left(A_{i} \cap L_{i j}^{\perp}\right) /\left(A_{i} \cap U_{i j}^{\perp}\right)
$$

is a section of the group $A_{i}$ for all $i, j \in I$. By condition (4), the intersection $S_{i j k}=S_{i j} \cap S_{i k}$ is not empty. Therefore $S_{i j k}=U_{i j k} / L_{i j k}$, where $U_{i j k}=U_{i j} \cap U_{i k}$ and $L_{i j k}=L_{i j} L_{i k}$. Thus the set

$$
A_{i j k}:=A_{i j} \cap A_{i k}=\left(\left(G_{i^{\prime}} U_{i j k}\right) /\left(G_{i^{\prime}} L_{i j k}\right)\right)^{\perp}
$$

is also not empty for all $i, j, k$. By condition (5), the isomorphism $f_{i j}$ takes $U_{i j k}$ and $L_{i j k}$ to, respectively, $U_{k i j}$ and $L_{k i j}$. It induces an isomorphism

$$
\tilde{f}_{i k}:\left(G_{i^{\prime}} U_{i j k}\right) /\left(G_{i^{\prime}} L_{i j k}\right) \rightarrow\left(G_{k^{\prime}} U_{k i j}\right) /\left(G_{k^{\prime}} L_{k i j}\right) .
$$

Set $g_{i k}=\widetilde{f}_{k i}^{\perp}$ for all $i, k \in I$. Then $g_{i k}\left(A_{i j k}\right)=A_{k i j}$ and $g_{i j k} g_{k i j} g_{j k i}=$ id for all $i, j, k \in I$. Thus, the triple

$$
(\mathfrak{G}, \mathfrak{S}, \mathfrak{F})^{\perp}:=\left(\mathfrak{G}^{\perp}, \mathfrak{S}^{\perp}, \mathfrak{F}^{\perp}\right)
$$

where $\mathfrak{G}^{\perp}=\left\{A_{i}\right\}_{i \in I}, \mathfrak{S}^{\perp}=\left\{A_{i j}\right\}_{i, j \in I}$, and $\mathfrak{F}^{\perp}=\left\{g_{i j}\right\}_{i, j \in I}$, satisfies conditions (4), (5) and (6). This proves the first part of the following statement (the second part follows by duality). 
Lemma 3.2. The triple (13) is a system of linked sections. Moreover,

$$
\left((\mathfrak{G}, \mathfrak{S}, \mathfrak{F})^{\perp}\right)^{\perp}=(\mathfrak{G}, \mathfrak{S}, \mathfrak{F}) \text { 色 }
$$

Note that if $(\mathfrak{G}, \mathfrak{S}, \mathfrak{F})$ is a system of linked quotients (respectively, subgroups), then $(\mathfrak{G}, \mathfrak{S}, \mathfrak{F})^{\perp}$ is a system of linked subgroups (respectively, quotients). This fact together with Lemma 3.2 immediately imply the following statement.

Corollary 3.3. For a fixed family $\mathfrak{G}$ of finite abelian groups, the mapping

$$
(\mathfrak{G}, \mathfrak{S}, \mathfrak{F}) \rightarrow(\mathfrak{G}, \mathfrak{S}, \mathfrak{F})^{\perp}
$$

induces a one-to-one correspondence between the systems of linked quotients based on $\mathfrak{G}$ and the systems of linked subgroups based on $\mathfrak{G}^{\perp}$.

\section{Quasiregular COHEREnt COnfigurations as Systems of Linked QUOTIENTS}

Throughout this section, $\mathcal{X}=(\Omega, S)$ is a quasiregular coherent configuration with fibers $\Omega_{1}, \ldots, \Omega_{m}, m \geq 1$, and $I=\{1, \ldots, m\}$. For each $i \in I$, the $i$ th homogeneous component $\mathcal{X}_{i}$ of $\mathcal{X}$ is a (regular) scheme the basis relations of which form a group $G_{i}$, and we say that $\mathcal{X}$ is of type $\mathfrak{G}=\left\{G_{i}\right\}_{i \in I}$. Note that any quasiregular coherent configuration is of type $\mathfrak{G}$ for suitable family of groups $G_{i} \in$ $\mathfrak{G}$. The aim of this section is to prove that a quasiregular coherent configuration of type $\mathfrak{G}$ is nothing else than a system of linked quotients based on $\mathfrak{G}$.

Let $i, j \in I$. In what follows, we set $S_{i j}:=S_{\Omega_{i}, \Omega_{j}}$; in particular, $S_{i i}=G_{i}$. Note that here, $S_{i j}$ is a subset of $S$ and not a section of a group as in Section 3. Since any relations from $G_{i}$ and $G_{j}$ are thin, we have

$$
s_{i}^{*} S_{i j}=S_{i j}=S_{i j} s_{j}, \quad s_{i} \in G_{i}, s_{j} \in G_{j} .
$$

This implies that the mappings $\pi_{i j}: G_{i} \rightarrow \operatorname{Sym}\left(S_{i j}\right)$ and $\rho_{i j}: G_{j} \rightarrow \operatorname{Sym}\left(S_{i j}\right)$ given by the formulas

$$
x^{\pi_{i j}(s)}=s^{*} x, x \in S_{i j}, \quad \text { and } \quad x^{\rho_{i j}(s)}=x s, x \in S_{i j},
$$

are group homomorphisms.

Lemma 4.1. In the above notation, for all $i, j \in I$,

(1) $\operatorname{im}\left(\pi_{i j}\right)$ and $\operatorname{im}\left(\rho_{i j}\right)$ are regular groups centralizing each other,

(2) $\operatorname{ker}\left(\pi_{i j}\right)=x x^{*}$ for all $x \in S_{i j}$, and $\operatorname{ker}\left(\rho_{i j}\right)=x^{*} x$ for all $x \in S_{j i}$.

Proof. Let $x, y \in S_{i j}$. Then any basis relations $s_{i} \in x y^{*}$ and $s_{j} \in x^{*} y$ belong to $G_{i}$ and $G_{j}$, respectively. Therefore they are thin and hence

$$
s_{i}^{*} x=y=x s_{j} .
$$

This proves that $\operatorname{im}\left(\pi_{i j}\right)$ and $\operatorname{im}\left(\rho_{i j}\right)$ are transitive groups. Obviously each of them centralizes the other. Next assume that $s_{i}^{*} x=x$ for some $x \in S_{i j}$. Since the group $\operatorname{im}\left(\rho_{j}\right)$ is transitive, any $y \in S_{i j}$ is of the form $x s_{j}$ for a suitable $s_{j}$. Therefore,

$$
s_{i}^{*} y=s_{i}^{*} x s_{j}=x s_{j}=y .
$$

Thus, the group $\operatorname{im}\left(\pi_{i j}\right)$ is regular. Similarly, one can prove that the group $\operatorname{im}\left(\rho_{i j}\right)$ is also regular. This proves statement (1). Now by the regularity, $s \in \operatorname{ker}\left(\pi_{i j}\right)$ if

\footnotetext{
${ }^{3}$ Here, we use canonical isomorphisms between $A_{i}^{\perp}$ and $G_{i}$, and between $A_{i j}^{\perp}$ and $G_{i j}$.
} 
and only if $s^{*} x=x$ for some (and hence for all) $x \in S_{i j}$ if and only if $s \in x x^{*}$. Similarly, $s \in \operatorname{ker}\left(\rho_{i j}\right)$ if and only if $s \in x^{*} x$ for some (and hence for all) $x \in S_{i j}$. $\square$

In what follows, we set

$$
G_{i j}=\operatorname{ker}\left(\pi_{i j}\right)=\operatorname{ker}\left(\rho_{j i}\right), \quad i, j \in I .
$$

From statement (1) of Lemma 4.1, it follows that the groups $G_{i} / G_{i j}$ and $G_{j} / G_{j i}$ are the left and right representations of the same group. In particular, these groups are isomorphic. To write an explicit isomorphism, choose arbitrarily points $\alpha_{i} \in \Omega_{i}$, $i \in I$, and set

$$
s_{i j}:=r\left(\alpha_{i}, \alpha_{j}\right), \quad i, j \in I .
$$

Lemma 4.2. For all $i, j \in I$, the mapping

$$
f_{i j}: G_{i} / G_{i j} \rightarrow G_{j} / G_{j i}, G_{i j} s_{i} \mapsto s_{i j}^{*}\left(G_{i j} s_{i}\right) s_{i j}
$$

is a group isomorphism, and $\left(f_{i j}\right)^{-1}=f_{j i}$.

Proof. To prove that $f_{i j}$ is well-defined, denote $s_{i j}$ by $x$. Then $s_{i} x=x s_{j}$ for some $s_{j} \in G_{j}$ and $G_{i j}=x x^{*}$ by statements (1) and (2) of Lemma 4.1, respectively. Thus, by Lemma 2.3 we have

$$
x^{*}\left(G_{i j} s_{i}\right) x=x^{*}\left(x x^{*}\right) s_{i} x=x^{*} s_{i} x=x^{*} x s_{j}=G_{j i} s_{j} \in G_{j} / G_{i j},
$$

which shows that $f_{i j}$ is well-defined. It is a homomorphism, because for any elements $s_{i}, t_{i} \in G_{i}$,

$$
f_{i j}\left(G_{i j} s_{i} G_{i j} t_{i}\right)=f_{i j}\left(G_{i j} s_{i} t_{i}\right)=x^{*}\left(s_{i} t_{i}\right) x=\left(x^{*} s_{i} x\right)\left(x^{*} t_{i} x\right)=f_{i j}\left(G_{i j} s_{i}\right) f_{i j}\left(G_{i j} t_{i}\right) .
$$

Assume that $f_{i j}\left(G_{i j} s_{i}\right)=G_{j i}$. Then formula (14) shows that $s_{j} \in G_{j i}$, and hence $s_{i} x=x s_{j} \in x x^{*} x=x$. It follows that $s_{i}^{*} \in G_{i j}$, and therefore $s_{i} \in G_{i j}$, which means that the homomorphism $f_{i j}$ is one-to-one. Formula (14) also shows that it is an epimorphism, because for every $s_{j} \in G_{j}$, there exists $s_{i} \in G_{i}$ such that $s_{i} x=x s_{j}$ (statement (1) of Lemma 4.1). Thus, $f_{i j}$ is an isomorphism. Since also

$$
f_{i j} f_{j i}\left(G_{j i} s_{i}\right)=f_{i j}\left(x s_{i} x^{*}\right)=x^{*} x s_{i} x^{*} x=G_{j i} s_{i},
$$

we conclude that $f_{i j} f_{j i}$ is the identity map.

At this point, starting with the quasiregular coherent configuration $\mathcal{X}$ with fibers indexed by the set $I$, we have constructed a triple

$$
\mathcal{T}=\mathcal{T}(\mathcal{X})=\left(\left\{G_{i}\right\}_{i \in I},\left\{G_{i} / G_{i j}\right\}_{i, j \in I},\left\{f_{i j}\right\}_{i, j \in I}\right) .
$$

Lemma 4.3. The triple $\mathcal{T}$ is a system of linked quotients.

Proof. To verify condition (5), let $i, j, k \in I$. From the definition of $s_{i j}$, it follows that $s_{j k} \in s_{j i} s_{i k}$. This implies that

$$
G_{j k}=s_{j k} s_{j k}^{*} \subseteq\left(s_{j i} s_{i k}\right)\left(s_{j i} s_{i k}\right)^{*}=s_{i j}^{*} s_{i k} s_{i k}^{*} s_{i j}=f_{i j}\left(G_{i k}\right),
$$

and similarly, $G_{i k} \subseteq f_{j i}\left(G_{j k}\right)$. Therefore, $f_{i j}\left(G_{i k}\right) \subseteq f_{i j} f_{j i}\left(G_{j k}\right)=G_{j i} G_{j k}$. Thus,

$$
G_{j i} G_{j k}=f_{i j}\left(G_{i k} G_{i j}\right) .
$$

This enables us to define the induced (by $f_{i j}$ ) isomorphism

$$
f_{i j k}: G_{i} / G_{i j} G_{i k} \rightarrow G_{j} / G_{j i} G_{j k} .
$$


It remains to verify condition (6), or, equivalently, that the composite $f_{k i j} f_{j k i} f_{i j k}$ is the identity map. To this end, let $x \in G_{i i}$. Then

$$
\begin{aligned}
f_{k i j} f_{j k i} f_{i j k}\left(G_{i j} G_{i k} x\right) & =f_{k i j} f_{j k i}\left(s_{j i} x s_{i j} G_{j k}\right) \\
& =f_{k i j}\left(s_{k j} s_{j i} x s_{i j} s_{j k}\right) \\
& =s_{i k} s_{k j} s_{j i} x s_{i j} s_{j k} s_{k i} .
\end{aligned}
$$

Note that $1_{\Omega_{i}} \in s_{i j} s_{j k} s_{k i} \cap s_{i k} s_{k j} s_{j i}$ by the definition of the relations $s_{i j}$. Therefore the left-hand side of the above equality equals the coset of $G_{i j} G_{i k}$ that contains $x$, and the right-hand side must be one coset, i.e., the $\operatorname{coset}\left(G_{i j} G_{i k} x\right)$, as required.

Let us show that every system of linked quotients based on a family $\mathfrak{G}$ of groups $G_{i}, i \in I$, is of the form $\mathcal{T}(\mathcal{X})$ for some quasiregular coherent configuration $\mathcal{X}$ of type $\mathfrak{G}$. To this end, assume that $I$ is a finite set and

$$
(\mathfrak{G}, \mathfrak{S}, \mathfrak{F})=\left(\left\{G_{i}\right\}_{i \in I},\left\{G_{i j}\right\}_{i, j \in I},\left\{f_{i j}\right\}_{i, j \in I}\right)
$$

is a system of linked quotients. Denote by $\Omega$ the disjoint union of all the $G_{i}$. For each $i \in I$ and each $x \in G_{i}$, we define a binary relation $s_{x}$ on the set $\Omega_{i}=G_{i}$ as the graph of the left multiplication by $x$,

$$
s_{x}=\left\{(\alpha, \beta) \in \Omega_{i} \times \Omega_{j}: \beta=x \alpha\right\} .
$$

Lemma 4.4. Given indices $i, j \in I$, the set $\Omega_{i} \times \Omega_{i}$ is partitioned into the relations $s_{x} \cdot s_{i j}, x \in G_{i}$, where

$$
s_{i j}=\bigcup_{g_{i} \in G_{i}}\left\{g_{i}\right\} \times f_{i j}\left(G_{i j} g_{i}\right) .
$$

Proof. We have to verify that if $x, y \in G_{i}$, then the relations $s_{x} \cdot s_{i j}$ and $s_{y} \cdot s_{i j}$ are disjoint or coincide. To this end, we note that

$$
s_{x} \cdot s_{i j}=\bigcup_{\gamma \in G_{i}}\{x \gamma\} \times f_{i j}\left(G_{i j} \gamma\right)=\bigcup_{\delta \in G_{i}}\{\delta\} \times f_{i j}\left(G_{i j} x^{-1} \delta\right) .
$$

Now if the relations $s_{x} \cdot s_{i j}$ and $s_{y} \cdot s_{i j}$ are intersected, say in a pair $(\alpha, \beta)$, then this implies that $\beta$ belongs to the intersection of two right cosets $f_{i j}\left(G_{i j} x^{-1} \alpha\right)$ and $f_{i j}\left(G_{i j}\left(y^{-1} \alpha\right)\right.$ of the same group $G_{j i} \leq G_{j}$. It follows that $G_{i j} x^{-1}=G_{i j} y^{-1}$ and hence $x=y z$ for some $z \in G_{i j}$. Since $z \cdot s_{i j}=s_{i j}$, we conclude that

$$
s_{x} \cdot s_{i j}=s_{y z} s_{i j}=s_{y} \cdot\left(s_{z} s_{i j}\right)=s_{y} \cdot s_{i j}
$$

as required.

Denote by $S$ the union of the sets $S_{i j}=\left\{s_{x} s_{i j} \mid x \in G_{i}\right\}$ over all $i, j \in I$.

Lemma 4.5. The pair $\mathcal{X}=(\Omega, S)$ is a quasiregular coherent configuration such that $\mathcal{T}(\mathcal{X})=(\mathfrak{G}, \mathfrak{S}, \mathfrak{F})$.

Proof. We have already proved that $S$ is a partition of $\Omega \times \Omega$. It is easily verified that $1_{\Omega}$ is a disjoint union of $s_{e_{i}}$ with $i \in I$, where $e_{i}$ is the identity of $G_{i}$. Next, let $i, j \in I$. By the definition of $s_{i j}$, we have

$$
s_{i j}^{*}=\bigcup_{\gamma \in G_{i}} f_{i j}\left(G_{i j} \gamma\right) \times\{\gamma\} .
$$

Take $(\alpha, \beta) \in s_{i j}^{*}$. Then $\beta \in f_{i j}\left(G_{i j} \alpha\right)$, which implies $f_{j i}\left(G_{j i} \beta\right)=G_{i j} \alpha$, because $\left(f_{i j}\right)^{-1}=f_{j i}$. Thus,

$$
(\beta, \alpha) \in\{\beta\} \times f_{j i}\left(G_{j i} \beta\right) \subseteq s_{j i}
$$


and, hence $s_{i j}^{*} \subseteq s_{j i}$. Since $i$ and $j$ were taken arbitrarily, it follows that $s_{j i}^{*} \subseteq s_{i j}$ and hence $s_{j i} \subseteq s_{i j}^{*}$. Thus, $s_{i j}^{*}=s_{j i}$ and so $S^{*}=S$.

We claim that $s_{i j} s_{j k}$ equals the union of all sets $s_{x} s_{i k}$ with $x \in G_{i j}$. To this end, let $(\alpha, \gamma) \in s_{i j} s_{j k}$. Then there exists $\beta \in G_{j}$ such that $(\alpha, \beta) \in s_{i j}$ and $(\beta, \gamma) \in s_{j k}$. In particular, we have $\beta \in f_{i j}\left(G_{i j} \alpha\right)$ and $\gamma \in f_{j k}\left(G_{j k} \beta\right)$. Since $G_{j k} G_{j i}=f_{i j}\left(G_{i j} G_{i k}\right)$ and $f_{j k i} f_{i j k} f_{k i j}=\mathrm{id}$, we conclude that

$$
\begin{aligned}
\gamma \in f_{j k}\left(G_{j k} f_{i j}\left(G_{i j} \alpha\right)\right) & =f_{j k}\left(f_{i j}\left(G_{i j} G_{i k}\right) f_{i j}\left(G_{i j} \alpha\right)\right) \\
& =f_{j k} f_{i j}\left(G_{i j} G_{i k} \alpha\right) \\
& =f_{k i}^{-1}\left(G_{i j} G_{i k} \alpha\right) \\
& =f_{i k}\left(G_{i j} G_{i k} \alpha\right) .
\end{aligned}
$$

This implies that $(\alpha x, \gamma) \in s_{i k}$ for some $x \in G_{i j}$, and hence,

$$
(\alpha, \gamma) \in s_{x} s_{i k} \subseteq \bigcup_{y \in G_{i j}} s_{y} s_{i k} .
$$

Thus, $s_{i j} s_{j k} \subseteq \bigcup_{x \in G_{i j}} s_{x} s_{i k}$. Conversely, if $x \in G_{i j}$, then

$$
s_{x} s_{i k} \subseteq s_{i j} s_{j i} s_{i k} \subseteq s_{i j} \bigcup_{y \in G_{j i}} s_{y} s_{j k}=s_{i j} s_{j k},
$$

which completes the proof of the claim.

It is easily verified that for each $x \in G_{i}$, there exists $y \in G_{j}$ such that $s_{x} s_{i j}=$ $s_{j i} s_{y}$. Furthermore, from the claim it follows that $s_{i j} \cdot s_{j k}$ is invariant with respect to the left multiplication by $s_{z}$ with $z \in G_{i j} G_{i k}$. Therefore the product of the adjacency matrices of $s_{x} s_{i j}$ and $s_{j k} s_{y}$ is a scalar multiple of the adjacency matrix of $s_{i j} \cdot s_{j k}$. This proves the existence of the intersection numbers for the partition $S$. Thus, $\mathcal{X}$ is a coherent configuration. The second statement of the lemma is straightforward.

Corollary 4.6. Let $\mathcal{X}$ be a quasiregular coherent configuration of type $\mathfrak{G}=\left\{G_{i}\right\}_{i \in I}$. Then there exist groups $G_{i j} \unlhd G_{i}, i, j \in I$, such that any basis relation $s \in S_{i j}$ is of the form

$$
s=\bigcup_{y \in G_{i}}\left\{x_{s} y\right\} \times f_{i j}\left(G_{i j} y\right) .
$$

for a suitable $x_{s} \in G_{i}$.

From Lemmas 4.3 and 4.5 , we immediately get the main result of this section.

Theorem 4.7. Let $\mathfrak{G}$ be a family of finite groups. Then the mapping $\mathcal{X} \rightarrow \mathcal{T}(\mathcal{X})$ gives a one-to-one correspondence between the quasiregular coherent configurations of type $\mathfrak{G}$ and the systems of linked quotients based on $\mathfrak{G}$.

As an example, let us consider a special case when all the groups of the family $\mathfrak{G}$ are simple. Then for all $i, j \in I$, the group $G_{i j}$ being a normal subgroup of $G_{i}$ is either $G_{i}$ itself or 1 . Moreover, if $G_{i j}=G_{j k}=1$ for some $k \in I$, then obviously $G_{i k}=1$. This implies that the relation on $I$, defined by

$$
i \sim j \quad \Leftrightarrow \quad G_{i j}=1,
$$

is an equivalence relation, and if $i \sim j$, then the groups $G_{i}$ and $G_{j}$ are isomorphic and $\left|S_{i j}\right|=\left|S_{j i}\right|=\left|G_{i}\right|=\left|G_{j}\right|$. Therefore, if $J$ is a class of $\sim$ and $\Omega_{J}$ is the union of 
all $\Omega_{i}$ with $i \in J$, then the coherent configuration $\mathcal{X}^{(J)}=\left(\Omega_{J}, S_{J}\right)$ is semiregular, where $S_{J}$ is the union of all $S_{i j}$ with $i, j \in J$. On the other hand,

$$
i \nsim j \Rightarrow S_{i j}=\left\{\Omega_{i} \times \Omega_{j}\right\}
$$

Therefore, the coherent configuration $\mathcal{X}$ is the direct sum of the coherent configurations $\mathcal{X}^{(J)}$, where $J$ runs over the classes of the equivalence relation $\sim$. According to [3, Theorem 3.3] each of these coherent configuration is schurian and separable. Since the direct sum of schurian (respectively, separable) coherent configurations is also schurian (respectively, separable) [3, Theorem 7.5], this proves the following statement.

Theorem 4.8. Let $\mathfrak{G}$ be a family of finite simple groups. Then any quasiregular coherent configuration $\mathcal{X}$ of type $\mathfrak{G}$ is the direct sum of semiregular coherent configurations. In particular, $\mathcal{X}$ is schurian and separable.

\section{Proof of Theorem 1.1}

Let $\mathfrak{G}=\left\{G_{i}\right\}_{i \in I}$, where $G_{i}$ is an abelian group and $I$ is a finite set. Then by Theorem 4.7 and Corollary 3.3 , the mapping

$$
\mathcal{X} \mapsto \mathcal{T}(\mathcal{X})^{\perp}
$$

defines a one-to-one correspondence between the quasiregular coherent configurations of type $\mathfrak{G}$ and the amalgam configurations based on $\mathfrak{G}$. This proves the first part of the theorem.

To prove the second part, let $\mathcal{X}=(\Omega, S)$ be a quasiregular coherent configuration of type $\mathfrak{G}$, and let $\mathcal{T}=\mathcal{T}(\mathcal{X})$ be the associated system of linked quotients defined by (15). Define the group

$$
G=\prod_{i \in I} G_{i}
$$

considered as a permutation group on $\Omega$ such that $\alpha s=\left\{\alpha^{s}\right\}$ for all $\alpha \in \Omega_{i}, s \in G_{i}$, and $i \in I$. In what follows, for each $i \in I$ we denote by $G_{i^{\prime}}$ the direct product of the groups $G_{j}$ with $j \neq i$.

Lemma 5.1. The coherent configuration $\mathcal{X}$ is schurian if and only if there exists a group $H \leq G$ such that for all $i, j \in I$,

$$
H^{\Omega_{i} \cup \Omega_{j}}=\left\{\left(s_{i}, s_{j}\right) \in G_{i} \times G_{j}: f_{i j}\left(G_{i j} s_{i}\right)=G_{j i} s_{j}\right\} .
$$

Proof. From Lemma 4.4, it follows that the condition given in the lemma is equivalent to the fact that $\operatorname{Orb}\left(H, \Omega_{i} \times \Omega_{j}\right)=S_{i j}$. Since the latter is true for all $i, j \in I$ if and only if $\mathcal{X}$ is schurian, we are done.

To complete the proof, we verify that if $\mathcal{X}$ is schurian, then the amalgam configuration $\mathcal{T}^{\perp}$ admits an amalgam (the converse statement can be proved in a similar way). To this end, assume that $\mathcal{X}$ is schurian. Then condition (16) is satisfied for some group $H \leq \operatorname{Sym}(\Omega)$; we take $H=\operatorname{Aut}(\mathcal{X})$. In what follows, assume that $\mathcal{T}^{\perp}$ is the amalgam configuration as in formula (13).

Let $i, j \in I$. If $i=j$, then condition (16) implies that $H^{\Omega_{i}}=G_{i}$, or equivalently, that $H G_{i^{\prime}}=G$. Passing to the dual subgroups, we get from this and the first equality in (9) that $A_{i} \cap H^{\perp}=1$ for all $i \in I$. Therefore if $\pi: A \rightarrow A / H^{\perp}$ is the canonical homomorphism, then

$$
\pi\left(A_{i}\right)=A_{i} H^{\perp}, \quad i \in I .
$$


Now assume that $i$ and $j$ are not necessarily equal. From condition (16), it follows that the group $H^{\Omega_{i} \cup \Omega_{j}}$ contains a subgroup $G_{i j} \times G_{j i}$. It follows that

$$
G_{i j} G_{i^{\prime}} \cap H=\left(\left(G_{i j} \times G_{j i}\right)\left(G_{i^{\prime}} \cap G_{j^{\prime}}\right)\right) \cap H=G_{j i} G_{j^{\prime}} \cap H .
$$

Note that in formula (12) written for the amalgam configuration $\mathcal{T}^{\perp}$, we should have $U_{i j}=G_{i}$ and $L_{i j}=G_{i j}$. Therefore, $A_{i j}=A_{i} \cap G_{i j}^{\perp}$ and $A_{j i}=A_{i} \cap G_{j i}^{\perp}$. It follows that passing to the dual subgroups in the left- and right-hand sides of the above equality, we have

$$
\pi\left(A_{i j}\right)=A_{i j} H^{\perp}=\left(A_{i} \cap G_{i j}^{\perp}\right) H^{\perp}=\left(A_{j} \cap G_{j i}^{\perp}\right) H^{\perp}=A_{j i} H^{\perp}=\pi\left(A_{j i}\right) .
$$

for all $i, j \in I$. Formulas (17) and (18) imply condition (7) in the definition of generalized free product of the groups $A_{i}$ with amalgamated subgroups $A_{i j}$. Thus, it remains to verify that the kernel $H^{\perp}$ of the homomorphism $\pi$ is generated by the elements $x^{-1} g_{i j}(x)$, where $x \in A_{i j}$ and $i, j \in I$. To this end, we note that that formula (16) shows that

$$
\operatorname{Aut}(\mathcal{X})=\bigcap_{i, j \in I} M_{i j}
$$

where $M_{i j}=\left\{\left(g_{i}\right)_{i \in I} \in G: f_{i j}\left(G_{i j} g_{i}\right)=G_{j i} g_{j}\right\}$. However, using the definitions of the operator $\perp$ and isomorphism $g_{i j}$, one can easily check that the group $M_{i j}^{\perp}$ consists of all elements $a^{-1} g_{i j}(a)$ with $a \in A_{i}$. Taking into account that

$$
\operatorname{Aut}(\mathcal{X})^{\perp}=\left\langle M_{i j}^{\perp}: i, j \in I\right\rangle
$$

(see formula (19)), we arrive to the required statement on the group $H^{\perp}=\operatorname{Aut}(\mathcal{X})^{\perp}$.

\section{Proof of Theorem 1.2}

In this section, we deduce Theorem 1.2 from a quite general result establishing schurity and separability for a large class of coherent configurations properly containing the class studied in [12. Namely, let $\mathcal{X}=(\Omega, S)$ be a coset t $^{4}$ coherent configuration, i.e., the following condition holds:

$$
s s^{*} s=s \text { for all } s \in S .
$$

From Lemma 2.3, it follows that every quasiregular coherent configuration is a coset one. We say that a coset coherent configuration $\mathcal{X}$ satisfies a distributivity condition if

$$
W \cap U V=(W \cap U)(W \cap V) \text { for all } U, V, W \in \mathcal{L}(\Delta), \Delta \in F,
$$

where $F=F(\mathcal{X})$ and $\mathcal{L}(\Delta)$ is the set of all intersections of closed subsets $s s^{*}$ with $s \in S_{\Delta, \Gamma}$ and $\Gamma \in F$. This condition obviously holds for any quasiregular coherent configuration whose homogeneous components correspond the groups with distributive lattice of normal subgroups.

Lemma 6.1. Let $\mathcal{X}$ be a coset coherent configuration satisfying the distributivity condition, and let $\varphi$ be an algebraic isomorphism from $\mathcal{X}$ to a coherent configuration $\mathcal{X}^{\prime}$. Then

(1) $\mathcal{X}^{\prime}$ is a coset coherent configuration satisfying the distributivity condition,

(2) each $\varphi$-faithful map is $\varphi$-extendable.

\footnotetext{
${ }^{4}$ The name comes from [5], where the coherent configurations admitted a regular abelian automorphism group and satisfying condition (20) were studied under that name.
} 
Proof. Statement (1) is obvious. To prove statement (2), let us verify that a $\varphi$ faithful map $f$ is $\varphi$-extendable to a point $\gamma \in \Omega$. Without loss of generality we assume that the set $\Lambda=\operatorname{Dom}(f)$ contains at least three distinct elements two of which, say $\alpha$ and $\beta$. Set

$$
\Lambda_{\alpha}=\Lambda \backslash\{\beta\} \quad \text { and } \quad \Lambda_{\beta}=\Lambda \backslash\{\alpha\}
$$

in particular, $\alpha \in \Lambda_{\alpha}$ and $\beta \in \Lambda_{\beta}$. By induction on $|\operatorname{Dom}(f)|$, we may assume that the restrictions $f_{\alpha}$ and $f_{\beta}$ of the map $f$ to, respectively, $\Lambda_{\alpha}$ and $\Lambda_{\beta}$ are $\varphi$-faithful maps. In view of formula (3), this implies that

$$
\bigcap_{\lambda \in \operatorname{Dom}\left(f_{\alpha}\right)} \lambda^{f_{\alpha}}\left(r_{\lambda \gamma}\right)^{\varphi} \neq \varnothing \text { and } \bigcap_{\lambda \in \operatorname{Dom}\left(f_{\beta}\right)} \lambda^{f_{\beta}}\left(r_{\lambda \gamma}\right)^{\varphi} \neq \varnothing
$$

where $r_{\lambda \gamma}=r(\lambda, \gamma)$. Take arbitrary elements $\alpha^{\prime}$ and $\beta^{\prime}$ belonging to the first and second intersection, respectively. Then for all $\lambda \in \Lambda_{\alpha} \cap \Lambda_{\beta}$, we have

$$
r^{\prime}\left(\alpha^{\prime}, \beta^{\prime}\right) \in\left(r_{\gamma \lambda}\right)^{\varphi}\left(r_{\lambda \gamma}\right)^{\varphi}=\left(r_{\gamma \lambda} r_{\lambda \gamma}\right)^{\varphi} \subseteq W^{\varphi}=: W^{\prime},
$$

where $W$ is the intersection of all sets $r_{\gamma \lambda} r_{\lambda \gamma}$ with $\lambda \in \Lambda_{\alpha} \cap \Lambda_{\beta}$. Note that since $\mathcal{X}$ is a coset coherent configuration, $W^{\prime}$ is a closed subset of $\mathcal{X}_{\Gamma^{\prime}}^{\prime}$, where $\Gamma$ is the fiber containing $\gamma$ and $\Gamma^{\prime}=\Gamma^{\varphi}$. Next,

$$
r^{\prime}\left(\alpha^{f}, \beta^{f}\right)=\left(r_{\alpha \beta}\right)^{\varphi} \in\left(r_{\alpha \gamma} r_{\gamma \beta}\right)^{\varphi}=\left(r_{\alpha \gamma}\right)^{\varphi}\left(r_{\gamma \beta}\right)^{\varphi} .
$$

Consequently, there exists a point $\gamma^{\prime} \in \Omega^{\prime}$ such that

$$
r^{\prime}\left(\alpha^{f}, \gamma^{\prime}\right)=\left(r_{\alpha \gamma}\right)^{\varphi} \quad \text { and } \quad r^{\prime}\left(\gamma^{\prime}, \beta^{f}\right)=\left(r_{\gamma \beta}\right)^{\varphi} .
$$

Furthermore, from the definition of $\alpha^{\prime}$ and formula (21) for $\lambda=\alpha$, we have $r^{\prime}\left(\alpha^{f}, \alpha^{\prime}\right)=\left(r_{\alpha \gamma}\right)^{\varphi}$. Therefore,

$$
r^{\prime}\left(\gamma^{\prime}, \alpha^{\prime}\right) \in r^{\prime}\left(\gamma^{\prime}, \alpha^{f}\right) r^{\prime}\left(\alpha^{f}, \alpha^{\prime}\right)=\left(r_{\gamma \alpha}\right)^{\varphi}\left(r_{\alpha \gamma}\right)^{\varphi}=\left(r_{\gamma \alpha} r_{\gamma \alpha}^{*}\right)^{\varphi}=: U^{\varphi},
$$

Similarly,

$$
r^{\prime}\left(\gamma^{\prime}, \beta^{\prime}\right) \in\left(r_{\gamma \beta} r_{\gamma \beta}^{*}\right)^{\varphi}=: V^{\varphi} .
$$

Again, $U^{\prime}=U^{\varphi}$ and $V^{\prime}=V^{\varphi}$ are closed subsets of the coherent configuration $\mathcal{X}_{\Gamma^{\prime}}^{\prime}$. Moreover,

$$
r^{\prime}\left(\gamma^{\prime}, \alpha^{\prime}\right) \in U^{\prime}, \quad r^{\prime}\left(\gamma^{\prime}, \beta^{\prime}\right) \in V^{\prime}, \quad r^{\prime}\left(\alpha^{\prime}, \beta^{\prime}\right) \in W^{\prime} .
$$

Since also $W^{\prime} \cap U^{\prime} V^{\prime}=\left(W^{\prime} \cap U^{\prime}\right)\left(W^{\prime} \cap V^{\prime}\right)$ (the distributivity condition), by the Zieschang lemma [12, Lemma 1] there exists a point $\bar{\gamma} \in \Omega^{\prime}$ such that

$$
\bar{\gamma} \in \alpha^{\prime}\left(W^{\prime} \cap U^{\prime}\right) \cap \beta^{\prime}\left(V^{\prime} \cap W^{\prime}\right) \cap \gamma^{\prime}\left(U^{\prime} \cap V^{\prime}\right) .
$$

To complete the proof, it suffices to verify that

$$
\bar{\gamma} \in \bigcap_{\lambda \in \Lambda} \lambda^{f}\left(r_{\lambda \gamma}\right)^{\varphi} .
$$

Since $r_{\alpha \gamma}(U \cap V)$ is contained in $r_{\alpha \gamma} r_{\alpha \gamma}^{*} r_{\alpha \gamma}=r_{\alpha \gamma}$, we have

$$
\left.r^{\prime}\left(\alpha^{f}, \bar{\gamma}\right) \in r^{\prime}\left(\alpha^{f},\right) i n p \gamma^{\prime}\right) r^{\prime}\left(\gamma^{\prime}, \bar{\gamma}\right)=\left(r_{\alpha \gamma}\right)^{\varphi}(U \cap V)^{\varphi}=\left(r_{\alpha \gamma}(U \cap V)\right)^{\varphi}=\left(r_{\alpha \gamma}\right)^{\varphi}
$$

and hence, $\bar{\gamma} \in \alpha^{f}\left(r_{\alpha \gamma}\right)^{\varphi}$. Similarly, we obtain $\bar{\gamma} \in \beta^{f}\left(r_{\beta \gamma}\right)^{\varphi}$. Finally for any $\lambda \in \Lambda_{\alpha} \cap \Lambda_{\beta}$, we have

$$
r^{\prime}\left(\lambda^{f}, \bar{\gamma}\right) \in r^{\prime}\left(\lambda^{f}, \beta^{\prime}\right) r^{\prime}\left(\beta^{\prime}, \bar{\gamma}\right) \subseteq\left(r_{\lambda \gamma}\right)^{\varphi} W^{\varphi} \subseteq\left(r_{\lambda \gamma} r_{\lambda \gamma}^{*} r_{\lambda \gamma}\right)^{\varphi}=\left(r_{\lambda \gamma}\right)^{\varphi}
$$

which proves (22). 
By the above remarks, Theorem 1.2 immediately follows from the theorem below, which is a straightforward consequence of Lemma 2.1 and 6.1.

Theorem 6.2. Any coset coherent configuration satisfying the distributivity condition is schurian and separable.

\section{Quasiregular COHEREnt COnfigurations With AT most FOUR FiBers}

Throughout this section, $\mathcal{X}=(\Omega, S)$ is a quasiregular coherent configuration with fibers $\Omega_{i}$ and $S_{i j}=S_{\Omega_{i}, \Omega_{j}}, i, j \in I$. First, we consider the case when $|I| \leq 3$.

Proof of Theorem 1.3. Let $\mathcal{X}^{\prime}=\left(\Omega^{\prime}, S^{\prime}\right)$ be a coherent configuration, and let

$$
\varphi: S \rightarrow S^{\prime}, s \rightarrow s^{\prime}
$$

be an algebraic isomorphism. It induces a bijection $\Omega_{i} \mapsto \Omega^{\prime}{ }_{i^{\prime}}$ between the fibers of $\mathcal{X}$ and $\mathcal{X}^{\prime}$, and an algebraic isomorphism from $\mathcal{X}_{\Omega_{i}}$ onto $\mathcal{X}_{\Omega_{i^{\prime}}^{\prime}}^{\prime}, i \in I$. In particular, the coherent configuration $\mathcal{X}^{\prime}$ is also quasiregular.

For each $i \in I$, we choose an arbitrary point $\alpha_{i} \in \Omega_{i}$, and set

$$
s_{i j}:=r\left(\alpha_{i}, \alpha_{j}\right), \quad i, j \in I .
$$

Then obviously $s_{i j} \in s_{i k} s_{k j}$, and hence $s_{i j}^{\prime} \in s_{i k}^{\prime} s_{k j}^{\prime}$ for all $i, j, k$. Since $|I| \leq 3$, there exist points $\alpha_{i}^{\prime} \in \Omega^{\prime}, i \in I$ such that

$$
s_{i j}^{\prime}=r\left(\alpha_{i}^{\prime}, \alpha_{j}^{\prime}\right), \quad i, j \in I .
$$

Furthermore, taking into account that the the coherent configurations $\mathcal{X}$ and $\mathcal{X}^{\prime}$ are quasiregular, we conclude that

$$
\Omega=\left\{\alpha_{i} s: s \in S_{i i}, i \in I\right\} \quad \text { and } \quad \Omega^{\prime}=\left\{\alpha_{i}^{\prime} s^{\prime}: s^{\prime} \in S_{i i}^{\prime}, i \in I\right\},
$$

where the singletons $\alpha_{i} s$ and $\alpha_{i}^{\prime} s^{\prime}$ are identified with the corresponding points of $\Omega_{i}$ and $\Omega_{i}^{\prime}$, respectively. It follows that the mapping

$$
f: \Omega \rightarrow \Omega^{\prime}, \alpha_{i} s \mapsto \alpha_{i}^{\prime} s^{\varphi},
$$

where $s \in S_{i i}$, is a bijection. We claim that it is a combinatorial isomorphism from $\mathcal{X}$ onto $\mathcal{X}^{\prime}$. Indeed, if $\alpha, \beta \in \Omega$, then in view of (23) there exist $s \in S_{i i}$ and $t \in S_{j j}$ such that $\alpha=\alpha_{i} s$ and $\beta=\alpha_{j}$. We have

$$
\begin{aligned}
r\left(\alpha^{f}, \beta^{f}\right) & =r\left(\alpha_{i}^{\prime} s^{\varphi}, \alpha_{j}^{\prime} t^{\varphi}\right) \\
& =\left(s^{\varphi}\right)^{*} r\left(\alpha_{i}^{\prime}, \alpha_{j}^{\prime}\right) t^{\varphi} \\
& =\left(s^{\varphi}\right)^{*}\left(s_{i j}\right)^{\varphi} t^{\varphi} \\
& =\left(s^{*} s_{i j} t\right)^{\varphi}=r(\alpha, \beta)^{\varphi} .
\end{aligned}
$$

Therefore the algebraic isomorphism $\varphi$ is induced by the combinatorial isomorphism $f$. Thus, the coherent configurations $\mathcal{X}$ is separable.

To prove the schurity of $\mathcal{X}$, let $r(\alpha, \beta)=r\left(\alpha^{\prime}, \beta^{\prime}\right)$, where $\alpha^{\prime}, \beta^{\prime} \in \Omega$. Assume first that $\alpha \in \Omega_{i}$ and $\beta \in \Omega_{j}$ with $i \neq j$. Then $(\alpha, \beta)^{f}=\left(\alpha^{\prime}, \beta^{\prime}\right)$, where $f$ is the isomorphism defined by (24) for $\varphi=\mathrm{id}_{S},\left(\alpha_{i}, \alpha_{j}\right)=(\alpha, \beta)$, and $\left(\alpha_{i}^{\prime}, \alpha_{j}^{\prime}\right)=\left(\alpha^{\prime}, \beta^{\prime}\right)$. It follows that for the group $K$ generated by these isomorphisms $f$ with all possible $(\alpha, \beta)$ and $\left(\alpha^{\prime}, \beta^{\prime}\right)$, we have

$$
\operatorname{Orb}\left(K, \Omega_{i} \times \Omega_{j}\right)=S_{i j}
$$


for all distinct $i$ and $j$. It immediately implies that if $|I| \neq 1$, then $K$ acts transitively on each $\Omega_{i}$, and hence $\mathcal{X}$ is schurian. Since the schurity of $\mathcal{X}$ for $|I|=1$ is obvious, we are done.

Proof of Corollary 1.4 Let $\mathcal{X}$ be a meta-thin scheme with thin residue of index at most 3. Denote by $\mathcal{Y}$ the thin residue extension of $\mathcal{X}$ in the sense of [9, Sec. 2.4]. Then $\mathcal{Y}$ is a quasiregular coherent configuration with at most 3 fibers (which are geometric cosets of the thin residue). By Theorem 1.3. it is schurian and separable. According to statement (3) in [9, Theorem 3.3], this shows that $\mathcal{X}$ is schurian and separable.

In the rest of the section, we construct a huge class of non-schurian quasiregular coherent configurations with exactly four fibers. To this end, let $G=C_{p} \times C_{p}$, where $p$ is a prime, and let $\mathfrak{G}=\left\{G_{i}\right\}_{i \in I}$, where $I=\{1,2,3,4\}$ and $G_{i}=G$ for all $i \in I$. Choose a family $\mathcal{G}$ of groups $G_{i j} \leq G_{i}, i, j \in I$, such that

$$
\left|G_{i j}\right|= \begin{cases}p & \text { if } i \neq j \\ 1 & \text { if } i=j .\end{cases}
$$

Then the factor groups $G_{i} / G_{i j}$ and $G_{j} / G_{j i}$ are isomorphic for all $i, j$. Therefore, one can choose a family $\mathfrak{F}$ of isomorphisms $f_{i j}: G_{i} / G_{i j} \rightarrow G_{j} / G_{j i}, i, j \in I$, such that

$$
f_{i i}=\operatorname{id}_{G} \quad \text { and } \quad f_{i j}=\left(f_{j i}\right)^{-1} .
$$

Assume, in addition, that

$$
G_{i j} \neq G_{i k} \text { for all } i, j, k \in I, j \neq k .
$$

In this case if the indices $i, j, k \in I$ are distinct then each of the sections $S_{i j k}=$ $G_{i} / G_{i j} G_{i k}$ and $S_{k i j}=G_{k} / G_{k i} G_{k j}$ is trivial and hence formulas (5) and (6) hold. The same is obviously true if some of $i, j, k$ are equal. Therefore the triple

$$
\mathcal{T}(\mathcal{G}, \mathfrak{F})=(\mathfrak{G}, \mathfrak{S}, \mathfrak{F}),
$$

where $\mathfrak{S}=\left\{G_{i} / G_{i j}\right\}_{i, j \in I}$ is a system of linked quotients. Denote by $\mathcal{X}(\mathcal{G}, \mathfrak{F})$ the quasiregular coherent configuration associated with the triple $\mathcal{T}(\mathcal{G}, \mathfrak{F})$.

Let us study the schurity question for a coherent configuration $\mathcal{X}=\mathcal{X}(\mathcal{G}, \mathfrak{F})$ for suitable families $\mathcal{G}$ and $\mathfrak{F}$. Let $a, i, j$ be distinct elements of $I$. Then condition (27) implies that

$$
G_{i a} \neq G_{i j} \quad \text { and } \quad G_{j a} \neq G_{j i}
$$

and hence

$$
G_{i a} \times G_{i j}=G=G_{j a} \times G_{j i} .
$$

It follows that the isomorphism $f_{i j a}: G_{i} / G_{i j} \rightarrow G_{j} / G_{j i}$ induces a unique isomorphism $\sigma_{i j a}: G_{i a} \rightarrow G_{j a}$ such that

$$
\left(x G_{i j}\right)^{f_{i j}}=x^{\sigma_{i j a}} G_{j i}, \quad x \in G_{i a} .
$$

Assume that the coherent configuration $\mathcal{X}$ is schurian. For each $i \in I$, choose a point $\alpha_{i} \in \Omega_{i}$. Denote by $k$ the index other than $a, i, j$, i.e., $\{a, i, j, k\}=\{1,2,3,4\}$. Then the sets

$$
\Omega_{i a}=\alpha_{i} G_{i a}, \quad \Omega_{j a}=\alpha_{j} G_{j a}, \quad \Omega_{k a}=\alpha_{k} G_{k a}
$$


are the neighborhoods of a point $\alpha_{a}$ in suitable basis relations of $\mathcal{X}$ (see Lemma 4.4). It follows that these sets are orbits of the group $\operatorname{Aut}(\mathcal{X})_{\alpha}$ for any $\alpha \in \Omega_{a}$. Moreover, for any two distinct indices $u, v \in\{i, j, k\}$, we have

$$
\left|r \cap\left(\Omega_{u a} \times \Omega_{v a}\right)\right|=p \quad \text { for all } r \in S_{u v},
$$

i.e., the relation $r_{u v}=r \cap\left(\Omega_{u a} \times \Omega_{v a}\right)$ is a matching. Note that $r_{u v} s_{v w} \in S_{u w}$ for all $r \in S_{u v}$ and $s \in S_{v w}$, where $w$ is the element of $\{i, j, k\}$ other than $u$ and $v$. This immediately implies that

$$
\sigma_{i j a} \sigma_{j k a} \sigma_{k i a}=\operatorname{id}_{G_{i}} .
$$

This proves the following statement.

Proposition 7.1. In the above notation, the coherent configuration $\mathcal{X}$ is schurian only if formula (29) holds true for all $i, j, k, a$ such that $\{i, j, k, a\}=\{1,2,3,4\}$.

There are many ways how to choose the families $\mathcal{G}$ and $\mathfrak{F}$ to satisfy the condition (29). To see a concrete example, let

$$
H_{1}=1 \times C_{p}, \quad H_{2}=C_{p} \times 1, \quad H_{3}=\operatorname{Diag}\left(C_{p} \times C_{p}\right) .
$$

Denote by $\mathcal{G}$ the family of the groups $G_{i j}$ that are $(i, j)$-entries of the following $4 \times 4$ array, $i, j \in I$ :

$$
\left(\begin{array}{cccc}
1 & H_{1} & H_{2} & H_{3} \\
H_{1} & 1 & H_{3} & H_{2} \\
H_{2} & H_{3} & 1 & H_{1} \\
H_{3} & H_{2} & H_{1} & 1
\end{array}\right) .
$$

In particular, $G_{i j}=G_{j i}$ for all $i$ and $j$. Next, each of the matrices

$$
\sigma_{23}=\left(\begin{array}{cc}
0 & 1 \\
1 & 0
\end{array}\right), \quad \sigma_{34}=\left(\begin{array}{cc}
1 & 0 \\
1 & 1
\end{array}\right), \quad \sigma_{42}=\left(\begin{array}{cc}
1 & -1 \\
0 & 1
\end{array}\right)
$$

with elements in $\mathbb{F}_{p}$, belongs to $\operatorname{GL}(2, p)$ and hence induces an automorphism of the group $G$ so that

$$
\left(H_{1}\right)^{\sigma_{23}}=H_{2}, \quad\left(H_{2}\right)^{\sigma_{34}}=H_{3}, \quad\left(H_{3}\right)^{\sigma_{42}}=H_{1} .
$$

Now, denote by $\mathfrak{F}$ the family the isomorphisms $f_{i j}: G / G_{i j} \rightarrow G / G_{j i}$ that are $(i, j)$-entries of the following $4 \times 4$ array, $i, j \in I$ :

$$
\left(\begin{array}{cccc}
\operatorname{id}_{G} & \operatorname{id}_{G / H_{1}} & \operatorname{id}_{G / H_{2}} & \operatorname{id}_{G / H_{3}} \\
\operatorname{id}_{G / H_{1}} & \operatorname{id}_{G} & f_{23} & f_{42}^{-1} \\
\operatorname{id}_{G / H_{2}} & f_{23}^{-1} & \operatorname{id}_{G} & f_{34} \\
\operatorname{id}_{G / H_{3}} & f_{42} & f_{34}^{-1} & \operatorname{id}_{G}
\end{array}\right),
$$

where the isomorphisms $f_{23}, f_{34}$, and $f_{42}$ are obtained from formula (28) for $a=1$ and $\sigma_{i j 1}=\left.\sigma_{i j}\right|_{H_{i-1}}$ with $(i, j)=(2,3),(3,4)$, and $(4,2)$, respectively. From the choice of the matrices $\sigma_{i j}$, it follows that

$$
\sigma_{23} \sigma_{34} \sigma_{42}=\left(\begin{array}{cc}
1 & 0 \\
1 & -1
\end{array}\right)
$$

which shows that condition (29) is not satisfied for $(a, i, j, k)=(1,2,3,4)$. Thus, Proposition 7.1 implies the following statement.

Proposition 7.2. For each prime $p$, the coherent configuration $\mathcal{X}(\mathcal{G}, \mathfrak{F})$ is not schurian. In particular, there are infinitely many non-schurian quasiregular coherent configurations with four fibers. 
We complete the section by a natural question, namely, whether the assumption in Proposition 7.1 gives a sufficient condition for the schurity of the coherent configuration $\mathcal{X}(\mathcal{G}, \mathfrak{F})$ ? It would also be interesting to find an analog of that proposition for the separability problem.

\section{REFERENCES}

[1] P. Dembowski and F. Piper, Quasiregular collineation groups of finite projective planes, Math. Z., 99, 53-75 (1967).

[2] S. Evdokimov and I. Ponomarenko, On highly closed cellular algebras and highly closed isomorphisms, Electronic J. Combin., 6, \#R18 (1999).

[3] S. Evdokimov and I. Ponomarenko, Permutation group approach to association schemes, European J. Combin., 30, No. 6, 1456-1476 (2009).

[4] S. Evdokimov and I. Ponomarenko, Schemes of a finite projective plane and their extensions, St. Petersburg Math. J., 21, No. 1, 65-93 (2010).

[5] S. Evdokimov and I. Ponomarenko, On coset closure of a circulant S-ring and schurity problem, J. Algebra Appl., 15, No. 4, Article ID 1650068 (2016).

[6] S. Friedland, Coherent algebras and the graph isomorphism problem, Discrete Appl. Math., 25, 73-98 (1989).

[7] D. G. Higman, Characterization of families of rank 3 permutation groups by the subdegree I, II, Arch. Math., 21, 151-156; 353-361 (1970).

[8] M. Hirasaka, On meta-thin association schemes, Des. Codes Cryptography, 34, 187-201 (2005).

[9] M. Muzychuk and I. Ponomarenko, On quasi-thin association schemes, J. Algebra, 351, 467-489 (2012)

[10] H. Neumann, Generalized free sums of cyclical groups, Amer. J. Math., 72, 671-685 (1950).

[11] P.-H. Zieschang, Theory of Association Schemes, Springer (2005).

[12] P.-H. Zieschang, Association schemes in which the thin residue is a finite cyclic group, J. Algebra, 324, No. 12, 3572-3578 (2010).

Department of Mathematics, Pusan National University, Jang-Jeon dong, Busan, RePUBLIC OF KOREA

E-mail address: hirasaka@pusan.ac.kr

Department of Mathematics, Pusan National University, Jang-Jeon dong, Busan, RePUBLIC OF KOREA

E-mail address: knukkj@pusan.ac.kr

St.Petersburg Department of the Steklov Mathematical Institute, St.Petersburg, RUSSIA

E-mail address: inp@pdmi.ras.ru 